\title{
Forecasting the Bandwidth of Digital Links to the Internet
}

\author{
Dimitry Bludov, Vladimir Bogdanov, Vitaly Golovko, Mikhail Simonov, Peter Vikhlyantsev \\ CentrInform, Russia
}

Correspondence: Dmitry Bludov, d.bludov@center-inform.ru

Manuscript communication: received 26 May 2012, accepted 5 July 2012

\begin{abstract}
In this paper, we propose a new efficient technique for forecasting the bandwidth of digital access links to the Internet. This technique can be used for calculating the throughput of access links in implementing electronic document management systems and in organizing information portals. The main idea is to estimate the bandwidth of digital access links to the Internet using the statistics of the service load. The main result is the derivation of analytical expressions to estimate the required bandwidth.
\end{abstract}

Keywords- Internet, bandwidth, digital highway access, virtual connections, data rate, denial of service probability.

\section{INTRODUCTION}

The number of services and/or functions provided in electronic form using information and communication technologies is increasing in modern society. The service provision system schedulers often need to ensure the required throughput of the digital access links to the Internet. The total traffic in the digital binding links is formed by the data flows which are generated by the participants (subscribers) in the exchange of electronic documents. An urgent problem in the current stage of system development is to forecast the incoming load and required throughput of the access links (Figure 1).

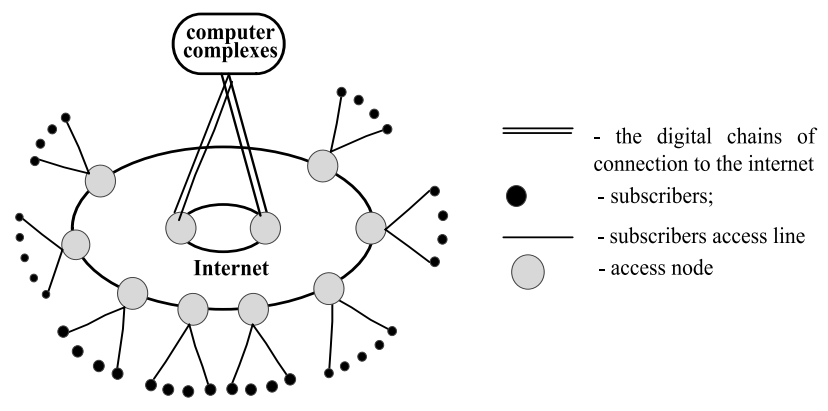

Figure 1. Internet access diagram.

In modern packet switching networks, which are constructed on the main IP/MPLS network towards the next generation communication networks (NGN) concept, the throughput is calculated based on the mass service system model. In [1], [2], the main attention is paid to calculating traditional network parameters, such as average service timeout, average packet queue length, and probability of timely packet delivery. However, the issues of subscriber access to the service provisioning with a given quality of service (QoS) have not been sufficiently studied.

This article describes the forecast of the throughput of the digital access links for a large number of subscribers, operating at different rates. The proposed methodology is published for the first time.

The article defines the quality of service parameters, the developed methodology of their estimate and examples of calculation, explaining the application of this methodology. In particular, Section 2 sets out indicators of bandwidth digital path and the main conditions and limitations. Section 3 shows the main conclusions of calculation expressions. Section 4 of the article shows examples of calculation.

\section{Throughput Parameters}

The following may be viewed as throughput parameters of the specific Internet digital access chain. Let $b$ and $B_{\text {en }}$ respectively denote the data transfer rate within the digital access link $u$ and the maximum implemented rate established by the Internet service provider. Due to the random pattern of load generation within the digital access link, the probability that $b$ does not exceed $B_{\mathrm{en}}$, denoted as $P\left(b \leq B_{\mathrm{en}}\right)$, may be viewed as a generalized parameter of throughput. The criterion of digital access link in compliance with the throughput requirements may then be given by

$$
P\left(b>B_{\text {en }}\right) \leq P_{\text {ref.add }},
$$

where $P_{\text {refadd }}$ is the permissible value of the denial of service probability.

Consider a scenario in which the server performance of a computing center allows to process the entire traffic coming from the Internet via the digital access chain. Assume that the computing center has $J$ digital connection chains to the Internet and $K$ classes of subscribers. The total number of subscribers in the $K$ traffic classes is given by $N=\sum_{k=1}^{K} N^{(k)}$, where $N^{(k)}$ is the number of subscribers in class $k$.

The load generated by each subscriber shall be described by the following conventional parameters for class $k$ : 
- $\gamma_{\text {sub }}^{(k)}$ : the Internet access intensity of the $k$-th class subscriber,

- $T_{\text {avg: }}^{(k)}$ the average duration of the data transfer session by the $k$-th class subscriber,

- $\eta_{\text {sub }}^{(k)}$ : the specific load intensity generated by the $k$-th class subscriber, defined as $\eta_{\text {sub }}^{(k)}=$ $\gamma_{\mathrm{sub}}^{(k)} T_{\mathrm{avg}}^{(k)} / 3600$.

According to [3], the data transfer rate of the $k$-th class subscriber shall be described by the following parameters:

- $B_{\text {avg }}^{(k)}, B_{\text {peak }}^{(k)}$ and $B_{\text {min }}^{(k)}$ : the average, peak and minimum data transfer rates, respectively,

- $K_{\mathrm{bf}}$ : the traffic batch feature, defined as $K_{\mathrm{bf}}=$ $B_{\text {peak }}^{(k)} / B_{\text {avg' }}^{(k)}$

- $T_{\text {peak }}^{(k)}$ : the average peak duration, which is the mean time of subscriber operation at the maximum data rate.

Let us also assume that the current data transfer rate of the $k$-th class subscriber, denoted by $b^{(k)}$, is a discrete random variable that may take on the peak value $B_{\text {peak }}^{(k)}$ with a probability of $p^{(k)}$ and the minimum value $B_{\text {min }}^{(k)}$ with a probability of $q^{(k)}=1-p^{(k)}$.

\section{General Expressions}

The probability density function (PDF) of the data transfer rate of the $k$-th class subscriber may be expressed in terms the $\delta$ function in the following way:

$$
f\left(b^{(k)}\right)=p^{(k)} \delta\left(b^{(k)}-B_{\text {peak }}^{(k)}\right)+q^{(k)} \delta\left(b^{(k)}+B_{\text {min }}^{(k)}\right) .
$$

Therefore, the first and the second initial moments are given by

$$
\begin{aligned}
& \beta_{1}^{(k)}=p^{(k)} B_{\text {peak }}^{(k)}+q^{(k)} B_{\text {min }}^{(k)} \\
& \beta_{2}^{(k)}=p^{(k)}\left[B_{\text {peak }}^{(k)}\right]^{2}-q^{(k)}\left[B_{\text {min }}^{(k)}\right]^{2}
\end{aligned}
$$

The mean of the data rate data is described by the first initial moment, i.e. $B_{\text {avg }}^{(k)}=\beta_{1}^{(k)}$. The variance of the data trasfer rate may be expressed in terms of the first and second initial moments as follows:

$$
D\left[b^{(k)}\right]=\beta_{2}^{(k)}+\left[\beta_{1}^{(k)}\right]^{2}=p^{(k)} q^{(k)}\left[B_{\text {peak }}^{(k)}-B_{\text {min }}^{(k)}\right]^{2} .
$$

For the purposes of forecasting the best estimate of the subscriber traffic, we shall approximate the subscriber operation by the traffic source of the "on-off" type.

In Figure 2(a), the data transfer of such a subscriber with constant data rate (CDR) is illustrated, while Figure 2(b) shows the variable data rate (VDR) within the session duration of $T_{\mathrm{c}}^{(k)}$; here, $B_{\min }=0$.

In paper [4], it is illustrated that this model allows to obtain the best estimate of the subscriber traffic. Under this model, the average value of the data transfer rate by the $k$-th class subscriber may be expressed as follows:

$$
B_{\text {avg }}^{(k)}=p^{(k)} B_{\text {peak }}^{(k)},
$$

where $p^{(k)}=1 / K_{\mathrm{bf}}$. Hence, we have

$$
\frac{1}{K_{\mathrm{bf}}}=\frac{B_{\text {peak }}^{(k)}}{B_{\text {avg }}^{(k)}}= \begin{cases}>1, & \text { for VDR source, } \\ 1, & \text { for CDR source. }\end{cases}
$$

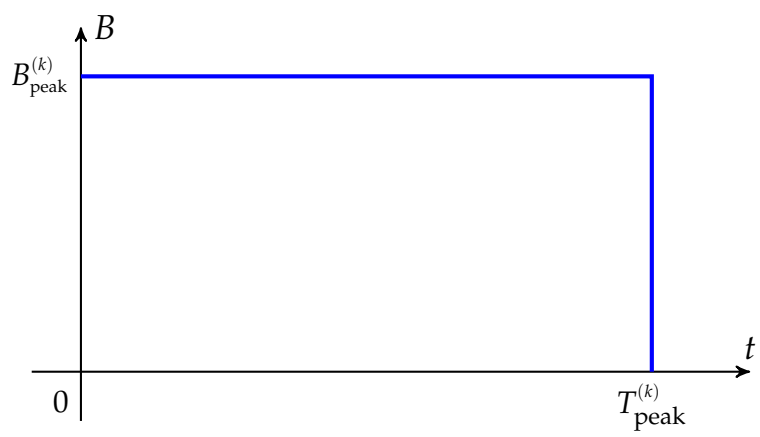

(a) Constant data rate

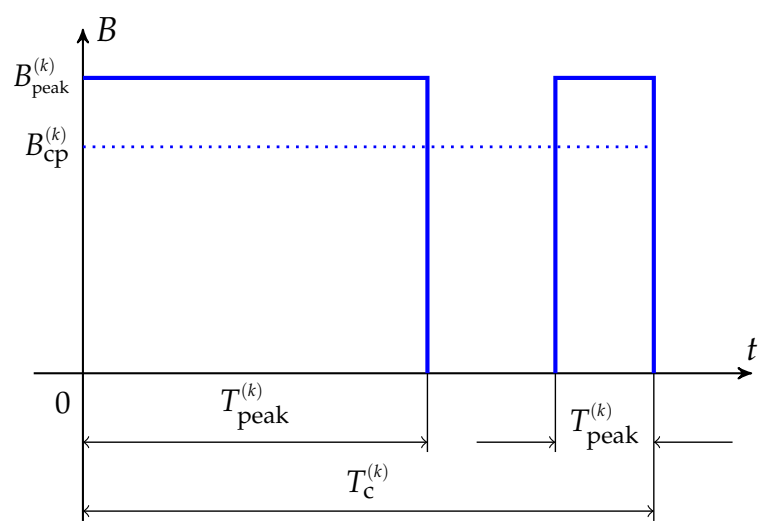

(b) Variable data rate

Figure 2. Variable subscriber data transfer rate.

The variance of the data transfer rate is then given by

$$
D\left[b^{(k)}\right]= \begin{cases}p^{(k)} q^{(k)}\left[B_{\text {peak }}^{(k)}\right]^{2}, & \text { for VDR source, } \\ 0, & \text { for CDR source. }\end{cases}
$$

Let us assume that the number of the virtual connections from all the $N^{(k)}$ subscribers of the $k$-th class to the Web-server of the computing center via the $j$-th access chain $\left(n_{\mathrm{vc}}^{(k)}\right)$ follows the Poisson distribution with mean of $N_{\mathrm{vc} . j}^{(k)}=\gamma_{\mathrm{vc}}^{(k)} T_{\text {avg }}^{(k)}$ and variance of $D\left[n_{\mathrm{vc}}^{(k)}\right]=N_{\mathrm{vc} . j}^{(k)}$. Here, the stream intensity of the virtual connection from the $k$-th class subscribers to the Web-server, $\gamma_{\mathrm{vc}}^{(k)}$, shall be given by $\gamma_{\mathrm{vc}}^{(k)}=N_{\mathrm{sub}}^{(k)} \gamma_{\mathrm{sub}}^{(k)}$.

The statistics of the data stream within the $j$-th access chain may be evaluated as the statistics of the sum of the subscriber data rates [5]. The mean and the variance of the random data stream rate within the $j$-th access chain $b_{i}^{(k)}$ from the $k$-th class subscribers can be found using the following expressions, respectively:

$$
\begin{aligned}
B_{\text {avg, }, j}^{(k)} & =N_{\text {avg }, j}^{(k)} B_{\text {avg }}^{(k)} \\
& =N_{\text {avg, } j}^{(k)} p^{(k)} B_{\text {peak }}^{(k)} . \\
D\left[b_{j}^{(k)}\right] & =\left[B_{\text {avg }}^{(k)}\right]^{2} D\left[N_{\mathrm{vc.j} j}^{(k)}\right]+N_{\mathrm{vc.j}}^{(k)} D\left[b^{(k)}\right] \\
& =N_{\mathrm{vc.j}}^{(k)} p^{(k)}\left[B_{\text {peak }}^{(k)}\right]^{2} .
\end{aligned}
$$

Due to the large amount of subscribers within each $k$ th class, the distribution of the data rate within the $j$ th access chain may be approximated by the normal distribution even in the case where the subscriber data rates follow arbitrary distribution laws. The general 
restriction imposed on the subscriber data rates consists in the fact that these should be more or less equal. This is most practically relevant to the single class subscribers, transferring formalized documents such as declarations. In this case the PDF of data transfer rate within the $j$-th access chain from all the $k$-th class subscribers shall be given by

$$
f\left(b_{j}^{(k)}\right)=\frac{1}{\sigma\left[b_{j}^{(k)}\right] \sqrt{2 \pi}} \exp \left[-\frac{\left[b_{j}^{(k)}-B_{\mathrm{avg}, j}^{(k)}\right]^{2}}{2 \sigma\left[b_{j}^{(k)}\right]^{2}}\right],
$$

where

$$
\begin{aligned}
\sigma\left[b_{j}^{(k)}\right] & =\sqrt{D\left[b_{j}^{(k)}\right]} \\
& =\sqrt{N_{\mathrm{vc} . j}^{(k)} p^{(k)}\left[B_{\text {peak }}^{(k)}\right]^{2}}
\end{aligned}
$$

is the standard deviation of the data rate within the $j$-th access chain from the $k$-th class subscribers.

It is well-known with the normal distribution law is that any linear combination of normal random variables is also normally distributed. Given that all the $k$-th class subscribers operate independently, the PDF of the data transfer rate $b_{j}$ within the $j$-th binding chain for data receiving from the subscribers of all the $K$ classes shall be expressed as

$$
f\left(b_{j}\right)=\frac{1}{\sigma\left[b_{j}\right] \sqrt{2 \pi}} \exp \left[-\frac{\left[b_{j}-B_{\mathrm{avg}, j}\right]^{2}}{2 \sigma\left[b_{j}\right]^{2}}\right],
$$

where

$$
\begin{aligned}
B_{\mathrm{avg}, j} & =\sum_{k=1}^{K} N_{\mathrm{vc.j}}^{(k)} p^{(k)} B_{\text {peak }}^{(k)}, \\
\sigma\left[b_{j}\right] & =\sqrt{\sum_{k=1}^{K} N_{\mathrm{vc.j}}^{(k)} p^{(k)}\left[B_{\text {peak }}^{(k)}\right]^{2} .}
\end{aligned}
$$

The probability of the event that the data transfer rate within the $j$-th binding digital chain does not exceed the maximum implemented is then given by

$P\left(b_{j} \leq B_{\mathrm{en}, j}\right)=\frac{1}{\sigma\left[b_{j}\right] \sqrt{2 \pi}} \int_{-\alpha}^{B_{\mathrm{en}, j}} \exp \left[-\frac{\left[b_{j}-B_{\mathrm{avg}, j}\right]^{2}}{2 \sigma\left[b_{j}\right]^{2}}\right] d b_{j}$

By replacing $\left(b_{j}-B_{\mathrm{avg}, j}\right) / \sigma\left[b_{j}\right]$ with $\lambda$, the above probability expression is reduced to

$$
\begin{aligned}
P\left(b_{j} \leq B_{\mathrm{en}, j}\right) & =\frac{1}{\sqrt{2 \pi}} \int_{-\alpha}^{\frac{B_{\mathrm{en}, j}-B_{\mathrm{avg}, j}}{\sigma\left[b_{j}\right]}} \exp \left(\frac{\lambda^{2}}{2}\right) d \lambda \\
& =F(u),
\end{aligned}
$$

where

$$
u=\left(B_{\mathrm{en}, j}-B_{\mathrm{avg}, j}\right) / \sigma\left[b_{j}\right]
$$

and $F(u)$ is the normal cummulative distribution function (CDF) of $u$. $F(u)$ function is tabulated or may be approximated to within $10^{-5}$ using the following expression [6]:

$$
P\left(b_{j} \leq B_{\mathrm{en}, j}\right)=\frac{1}{\sqrt{2 \pi}}\left(a_{1} x+a_{2} x^{2}+a_{3} x^{3}\right) e^{-\frac{u^{2}}{2}},
$$

Table I

Denial of Service Probability Values

\begin{tabular}{cc}
\hline$P_{\text {ref.add }}$ & $u$ \\
\hline \hline $10^{-2}$ & -2.326 \\
\hline $10^{-3}$ & -3.090 \\
\hline $10^{-4}$ & -3.719 \\
\hline $10^{-5}$ & -4.265 \\
\hline $10^{-6}$ & -4.753 \\
\hline $10^{-7}$ & -5.199 \\
\hline $10^{-8}$ & -5.612 \\
\hline $10^{-9}$ & -5.998 \\
\hline $10^{-10}$ & -6.631 \\
\hline
\end{tabular}

where $x=1 /(1+c u)$ with $c=0,33267$, and the constants $a_{1}, a_{2}$ and $a_{3}$ are $0.4361836,-0.1201676$ and 0.9372980 , respectively.

At known values of the data transfer rate expectation

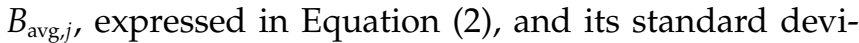
ation $\sigma\left[b_{j}\right]$, expressed in (3), and the permissible value of the top margin of the denial of service probability $P_{\text {add }}$, the required data rate of the $j$-th binding digital chain $B_{\mathrm{en}, j}$ can be forecasted by

$$
B_{\mathrm{en}, j}=B_{\mathrm{avg}, j}+u \sigma\left[b_{j}\right]
$$

where, for different referenced denial of service probability values, $P_{\text {ref.add, }}$, the random variable $u$ shall be given in Table I.

If the entire capacity of the $j$-th binding digital access chain is used for servicing the subscribers of a single class $k$ (with its associated parameters $p^{(k)}$ and $\left.B_{\text {peak }}^{(k)}\right)$, such as for receiving the declarations from the subscribers, then the maximum number of the virtual connections, which may be simultaneously serviced at the $j$-th access chain with the required QoS, can be obtained by

$$
N_{\mathrm{vc} . j \max }^{(k)}=\frac{B_{\mathrm{en}, j}}{p^{(k)} B_{\mathrm{peak}}^{(k)}}-\frac{u^{2}}{2 p^{(k)}}\left[\sqrt{1+4 \frac{B_{\mathrm{en}, j}}{u^{2} B_{\text {peak }}^{(k)}}}-1\right] .
$$

If the entire capacity of the $j$-th chain is used for transferring data by the subscribers of classes 1 and 2 (with parameters $p^{(1)}, B_{\text {peak }}^{(1)}$ and $\left.p^{(2)}, B_{\text {peak }}^{(2)}\right)$, then the number of the virtual connections at a given top margin of the denial of service probability for class 1 and class 2 can be given by Equations (9) and (10) at the top of the next page.

Generally, the entire capacity of the $j$-th binding digital access chain is used for transferring data by the subscribers of all $K$ classes. Then, by mathematical induction technique, the estimated number of the virtual connections for class $i$ is given by Equation (11), for $i=1, \ldots, K$.

\section{Sample Calculations}

\subsection{Example 1}

We want to calculate the number of the virtual connections of the subscribers to the Web-server of the computing center, given the following conditions: 


$$
\begin{aligned}
& N_{\mathrm{vc}, j}^{(1)}=\frac{B_{\mathrm{en}, j}-N_{\mathrm{vc}, j}^{(2)} p^{(2)} B_{\text {peak }}^{(2)}}{p^{(1)} B_{\text {peak }}^{(1)}}-\frac{u^{2}}{2 p^{(1)}}\left[\sqrt{1+4 \frac{B_{\mathrm{en}, j}-N_{\mathrm{vc}, j}^{(2)} p^{(2)} B_{\text {peak }}^{(2)}\left(1-B_{\text {peak }}^{(2)} / B_{\text {peak }}^{(1)}\right)}{u^{2} B_{\text {peak }}^{(1)}}}-1\right] \\
& N_{\mathrm{vc} . j}^{(2)}=\frac{B_{\mathrm{en}, j}-N_{\mathrm{vc.j}}^{(1)} p^{(1)} B_{\text {peak }}^{(1)}}{p^{(2)} B_{\text {peak }}^{(2)}}-\frac{u^{2}}{2 p^{(2)}}\left[\sqrt{1+4 \frac{B_{\mathrm{en}, j}-N_{\mathrm{vccj}}^{(1)} p^{(1)} B_{\text {peak }}^{(1)}\left(1-B_{\text {peak }}^{(1)} / B_{\text {peak }}^{(2)}\right)}{u^{2} B_{\text {peak }}^{(2)}}}-1\right] \\
& N_{\mathrm{vc}, j}^{(i)}=\frac{B_{\mathrm{en}, j}-\sum_{\forall k \neq i} N_{\mathrm{vc} . j}^{(k)} p^{(k)} B_{\text {peak }}^{(k)}}{p^{(i)} B_{\text {peak }}^{(i)}}-\frac{u^{2}}{2 p^{(i)}}\left[\sqrt{1+4 \frac{B_{\mathrm{en}, j}-\sum_{\forall k \neq i} N_{\mathrm{vc} . j}^{(k)} p^{(k)} B_{\text {peak }}^{(k)}\left(1-B_{\text {peak }}^{(k)} / B_{\text {peak }}^{(i)}\right)}{u^{2} B_{\text {peak }}^{(i)}}}-1\right] .
\end{aligned}
$$

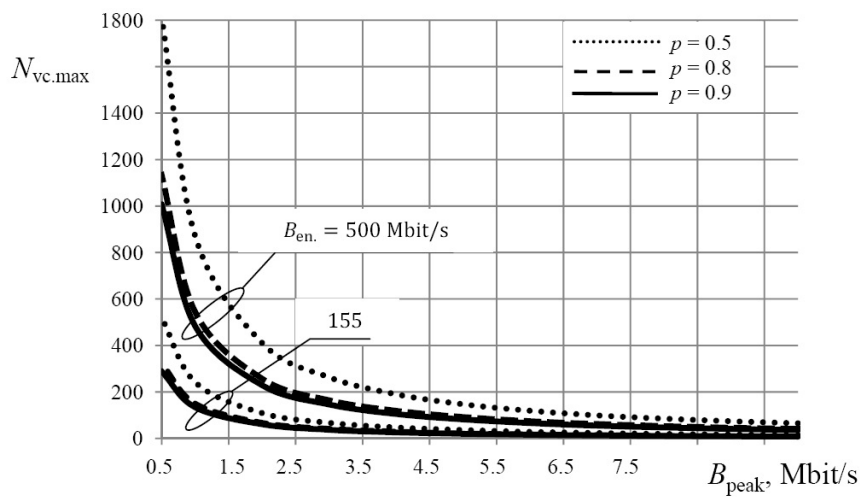

Figure 3. Maximum permissible number of virtual connections from the subscribers to the computing center at data transfer rates of 500 and $155 \mathrm{Mb} / \mathrm{s}$, for three different values of $p(0.9,0.8$ and 0.5$)$.

- The data transfer rate at the $j$-th digital access chain, $B_{\text {en }, j}$, are evaluated at three different values 155,300 and $500 \mathrm{Mb} / \mathrm{s}$;

- The denial of service probability via the digital access chain, $P\left(b_{j}>B_{\mathrm{en}, j}\right)$, shall not exceed $10^{-3}$. The argument of the CDF of the normal distribution is $u=3.08$, relevant to this probability;

- The subscriber operation parameters in three classes are as follows: $B_{\text {peak }}^{(1)}=64 \mathrm{~Kb} / \mathrm{s}, B_{\text {peak }}^{(2)}=$ $512 \mathrm{~Kb} / \mathrm{s}, B_{\text {peak }}^{(3)}=2 \mathrm{Mb} / \mathrm{s}, p^{(1)}=0.9, p^{(2)}=0.8$, $p^{(3)}=0.5$;

- The digital access chain is used for receiving the data from the single-class subscribers only.

Using expression (8) at access rate of $155 \mathrm{Mb} / \mathrm{s}$, we obtain the maximum possible number of virtual connections to the Web-server of the computing center of the subscribers of the single-class 1,2 and 3 as: $N_{\mathrm{vc}, \mathrm{j} \max }^{(1)}=2590, N_{\mathrm{vc.j} \max }^{(2)}=325$, and $N_{\mathrm{vc.j} \text { max }}^{(3)}=109$, respectively.

Figure 3 illustrates the dependency of the maximum permissible number of virtual connections from the subscribers to the computing center at three probability values of $p(0.9,0.8$ and 0.5$)$ subscriber operation at peak rate $B_{\text {peak }}^{(k)}$ and with two data transfer rates $\left(B_{\text {en }, j}=\right.$ 500 and $155 \mathrm{Mb} / \mathrm{s}$ ) within the digital access chain.

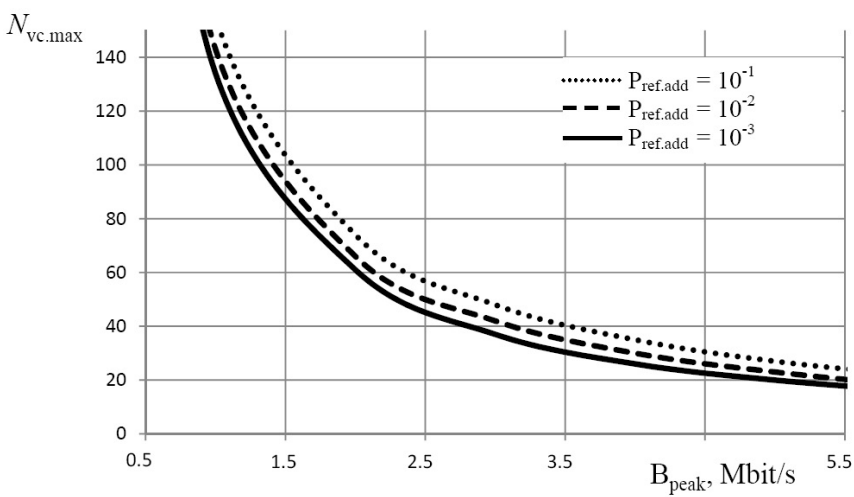

Figure 4. Dependency of the maximum number of permissible virtual connections from the subscribers to the computing center at $p=0.9$.

It can be seen that the number of virtual connections increases with the decrease of the subscriber access rate and with the growth of data transfer within the access chain of the computing center. Especially a sharp growth is seen at the subscriber access rates below $1.5 \mathrm{Mb} / \mathrm{s}$.

The effect of the permissible denial of service probability with respect to the maximum possible number of virtual connections is shown in Figure 4. The curves are calculated for the probability of $p=0.9$ and the peak data rate. The figure shows an insignificant effect of the denial of service probability $P_{\text {add }}$ on the number of virtual connections. Thus, an increase of the denial of service probability from $10^{-3}$ to $10^{-1}$ only causes the number of virtual connections to increase by no more than 10.

Using expression (11), we now calculate the numbers of virtual connections to the computing center from the subscribers of two classes, $N_{\mathrm{vc.j}}^{(1)}$ and $N_{\mathrm{vc} . j}^{(2)}$. Given that the denial of service probability is below $10^{-3}$, Figure 5 plots $N_{\mathrm{vc}, j}^{(1)}$ and $N_{\mathrm{vc} . j}^{(2)}$, for data transfer rates of 155,300 and $500 \mathrm{Mb} / \mathrm{s}$. The curves indicate that, with $N_{\mathrm{vc} . j}^{(1)}=$ $1000, N_{\mathrm{vc} . j}^{(2)}$ shall not exceed 196, 529 or 1000 if the data transfer rate is $155,300 \mathrm{Mb} / \mathrm{s}$ or $500 \mathrm{Mb} / \mathrm{s}$, respectively. 


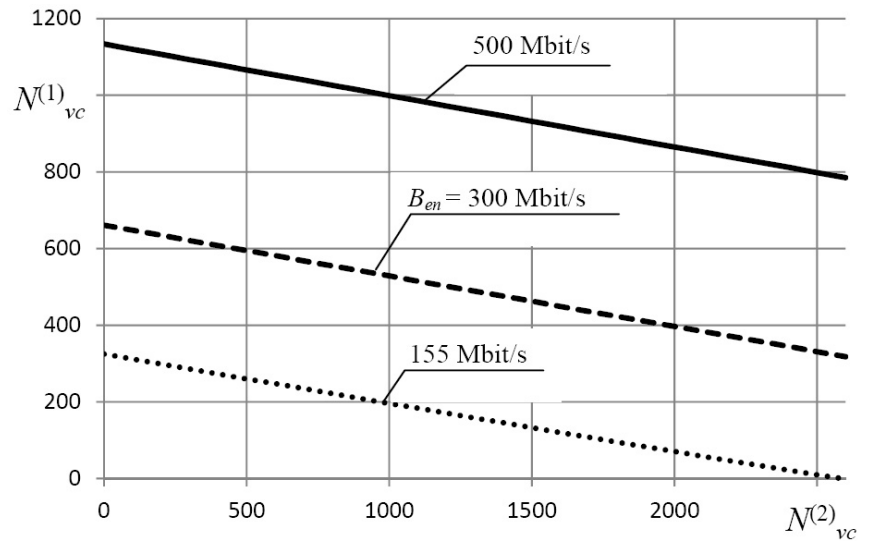

Figure 5. Numbers of virtual connections to the computing center from the subscribers of two classes at denial of service probability below $10^{-3}: N_{\mathrm{vc.j}}^{(1)}$ for class 1 with $B_{\text {peak }}^{(1)}=64 \mathrm{~Kb} / \mathrm{s}, p^{(1)}=0.9$, and $N_{\mathrm{vc.j}}^{(2)}$ for class 2 with $B_{\text {peak }}^{(2)}=512 \mathrm{~Kb} / \mathrm{s}, p^{(2)}=0.8$.

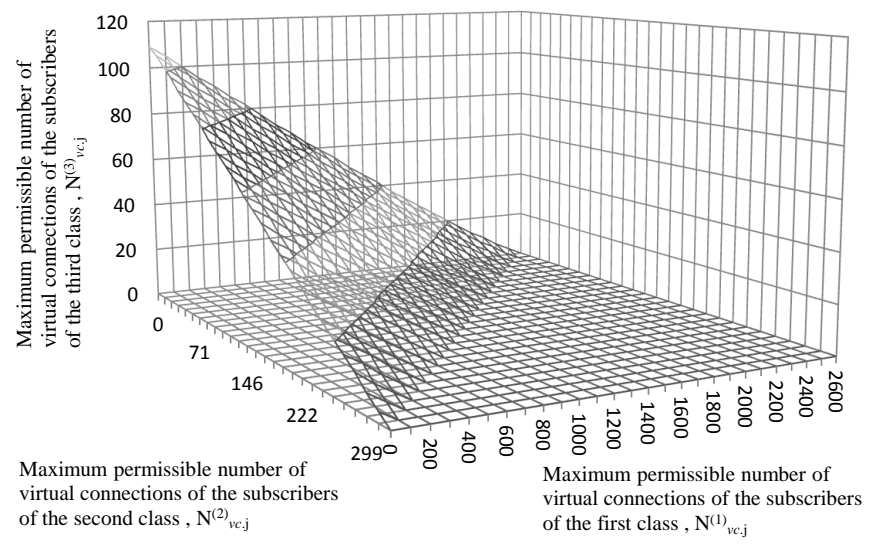

Figure 6. Number of virtual connections with the computing center of the subscribers of the first, second and third classes.

Number of virtual connections with the computing center of the first and third class subscribers.

The character of dependency of the number of virtual connections from the access chain data rate of the computing center shall be similar to illustrated in Figure 4.

Thus, at $N_{\mathrm{vcj}}^{(1)}=1000$ the number of the virtual connections with the computing center of the third class subscribers shall not exceed $N_{\mathrm{vcj}}^{(3)}=63$ at data rate within the access chain at $155 \mathrm{Mb} / \mathrm{s}$ and shall not exceed 184 virtual connections at $300 \mathrm{Mb} / \mathrm{s}$ and shall not exceed 361 at $500 \mathrm{Mb} / \mathrm{s}$.

If the digital access chain with data rate at $155 \mathrm{Mb} / \mathrm{s}$ is used for the receiving of the data from the subscribers of the three classes and the number of connections from the two classes amounts to $N_{\mathrm{vcj}}^{(1)}=1000$ and $N_{\mathrm{vcj}}^{(2)}=100$, then under expression (11) permissible number of the virtual connections of the third class subscribers shall not exceed $N_{\mathrm{vcj}}^{(3)}=30$, and at rates 300 and $500 \mathrm{Mb} / \mathrm{s}$ this shall not exceed 148 and 324 virtual connections respectively.

The results of the calculations for the three classes of subscribers and digital access chain data rate at 155 $\mathrm{Mb} / \mathrm{s}$ are given in figure 6 as the surface, limiting the maximum number of virtual connections under above conditions.

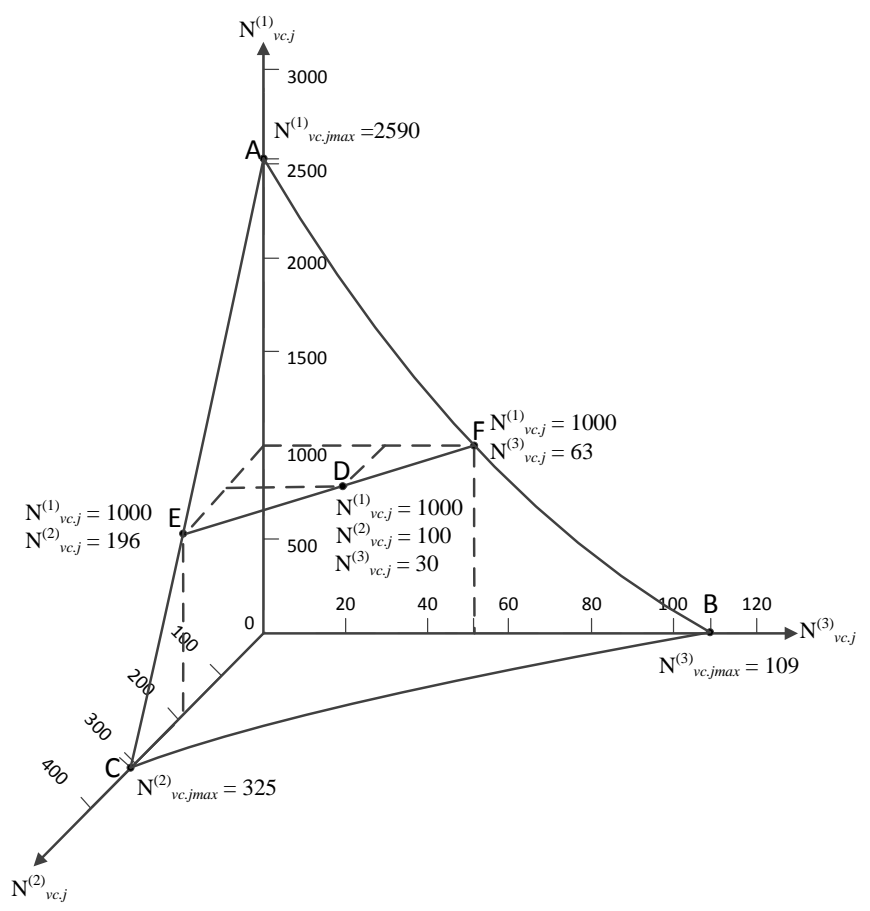

Figure 7. Dependency of the maximum permissible number of the virtual connections with the computing center of the subscribers of all the classes at their joint operation.

Figure 7 shows the maximum permissible number of virtual connections of the subscribers of the three classes $N_{\text {vcj.max }}^{(1)}=2590, N_{\text {vcj.max }}^{(2)}=325, N_{\text {vcj.max }}^{(3)}=109$, which corresponds the relevant points $A, B$ and $C$ in the plot.

Point E corresponds the number of virtual connections $N_{\mathrm{vcj} \cdot \max }^{(1)}=1000, N_{\mathrm{vci} \cdot \max }^{(2)}=196$ at joint operation of the subscribers of the first and second classes, and point $\mathrm{F}$ corresponds $N_{\mathrm{vcj} \cdot \max }^{(1)}=1000, N_{\mathrm{vcj} \cdot \max }^{(3)}=63$ at joint operation of the subscribers of the first and third classes.

Point $\mathrm{D}$ at the surface of the 3D-diagram shows the number of the virtual connections of subscribers $N_{\text {vcj.max }}^{(1)}=1000, N_{\text {vcj.max }}^{(1)}=100, N_{\text {vcj.max }}^{(1)}=30$ of each class at their joint operation.

Therefore, by the number of the virtual connections of the each class subscribers to the computing center we may evaluate the sufficiency of the digital binding chain throughput at compliance of the established requirements to the denial of service probability in the maximum load time.

\subsection{Example 2}

Define the probability of denial to receive the declaration due to the limited throughput of the digital binding chain of the computing center at the conditions below:

- The digital binding chain provides the data transfer rate at $B_{\mathrm{en}}=100 \mathrm{Mb} / \mathrm{s}$;

- The operation of the applicants of the three classes is described by the following parameters: $N_{\mathrm{vc}}^{(1)}=$ $300, p^{(1)}=0,9, B_{\text {peak }}^{(1)}=512 \mathrm{~Kb} / \mathrm{s} ; N_{\mathrm{vc}}^{(2)}=46, p^{(2)}=$ $0,8, B_{\text {peak }}^{(2)}=1 \mathrm{Mb} / \mathrm{s} ; N_{\mathrm{vc}}^{(3)}=78, p^{(3)}=0,5, B_{\mathrm{p}}^{(3)}=2$ $\mathrm{Mb} / \mathrm{s}$. 
- The digital binding chain is used to receive declarations only.

Based on (2) we define the expectation value of the data rate within the binding digital chain $B_{\text {avg }}=$ $\sum_{k=1}^{3} n_{\mathrm{vc}}^{(k)} p^{(k)} B_{\text {peak }}^{(k)}=80,64 \mathrm{Mb} / \mathrm{s}$.

Based on (3) we define the data rate standard deviation $\sigma[b]=\sqrt{\sum_{k=1}^{3} n_{\mathrm{vc}}^{(k)} p^{(k)}\left[B_{\text {peak }}^{(k)}\right]^{2}}=7,89$.

Based on (5) we calculate the argument of the normal distribution function $u=\left(B_{\mathrm{en}}-B_{\mathrm{avg}}\right) / \sigma[b]=2,45$.

With consideration of (6) the declaration receiving denial probability amounts to $P\left(b>B_{\mathrm{en}}\right)=1-P(b \leq$ $\left.B_{\text {en }}\right)=7,2 \cdot 10^{-3}$.

\subsection{Example 3}

Define the required data rate of the digital Internet access chain of the computing center to receive the electronic declaration based on the following initial data:

- The denial of service probability in receiving the declaration due to the limited throughput of the digital binding chain of the computing center shall not exceed $10^{-3}$ (argument of the normal distribution function $u=-3,08$ );

- The operation of the three classes of applicants is described by the following parameters: $N_{\mathrm{vc}}^{(1)}=500$, $p^{(1)}=0,9, B_{\text {peak }}^{(1)}=64 \mathrm{~Kb} / \mathrm{s} ; N_{\mathrm{vc}}^{(2)}=120, p^{(2)}=0,8$, $B_{\text {peak }}^{(2)}=512 \mathrm{~Kb} / \mathrm{s} ; N_{\mathrm{vc}}^{(3)}=50, p^{(3)}=0,5, B_{\mathrm{p}}^{(3)}=2$ $\mathrm{Mb} / \mathrm{s}$.

- The digital binding chain is used to receive declarations only.

Based on (2) we define the expectation value of the data rate within the binding digital chain $B_{\text {avg }}=$ $\sum_{k=1}^{3} N_{\mathrm{vc}}^{(k)} p^{(k)} B_{\text {peak }}^{(k)}=126,1 \mathrm{Mb} / \mathrm{s}$.

Based on (3) we define the data rate standard deviation $\sigma[b]=\sqrt{\sum_{k=1}^{3} N_{\mathrm{vc}}^{(k)} p^{(k)}\left[B_{\text {peak }}^{(k)}\right]^{2}}=11,21$. The required data rate of the digital access chain shall be at least $B_{\mathrm{en}}=B_{\mathrm{avg}}-u \sigma[b]=91,4 \mathrm{Mb} / \mathrm{s}$.

\section{Conclusion}

Using some relatively general assumptions on the subscriber data transfer rates, this paper proposes a technique to estimate the client denial of service during the maximum load times due to the limited data transfer rates within the Internet access digital chain; also the necessary throughput can be evaluated in order to satisfy given requirements.

Given any denial of service probability during the maximum load time, this technique can also be used to estimate the amount of the required data transfer rates within the Internet access digital chain of the computer center and the number of the maximum possible virtual connections of the subscribers.

Finally, based on the proposed technique, we can estimate the throughput of the access chains in implementing electronic document management systems and in organizing information portals. This would be very useful to set up an electronic organization for governmental services.

\section{REFERENCES}

[1] A. N. Nazarov and K. I. Sychev, Models and Methods of Calculation of Operation Quality Parameters for Node Equipment and Structural and Network Parameters of Next Generation Communication Networks. Krasnoyarsk: Polycom, 2010, (in Russian).

[2] D. S. Gulevich, Next Generation Communication Networks. Binompress, 2007, (in Russian).

[3] ITU-T, B-ISDN general network aspects, ITU-T Std. Recommendation I.311, 1992.

[4] E. S. Ventzel and P. A. Ovcharov, Probability Theory and Its Engineering Implications. Vysshaya Shkola, 2000, (in Russian).

[5] A. N. Nazarov and M. V. Simonov, ATM - High-Speed Data Network Technologies. Eco-Trends, 1997.

[6] M. Abramovitz and I. Stigan, Eds., Handbook on the Special Functions. Nauka, 1979, (in Russian).

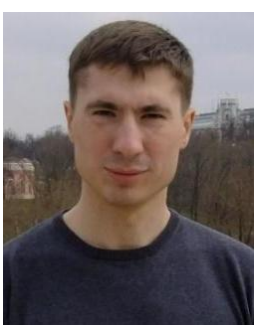

Dimitry Bludov is the Chief Specialist of Advanced Information Technology FSUE "CentrInform". In 2005, he was qualified as an engineer in the specialty "Automation of technological processes and production" at Kurgan State University. For several years, he led the IT department at a large trading company in the city of Kurgan. He joined the Federal State Unitary Enterprise "CentrInform" in early 2011. Dr. Bludov has participated in several state projects.

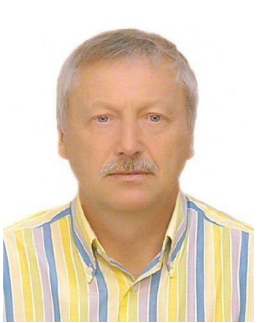

Vladimir Bogdanov is the General Director of FSUE "CentrInform". He graduated from the Leningrad Institute of Railway Engineers in 1980. During 1996 to 2009, he headed the branch of STC "Atlas" in St. Petersburg and Leningrad region (St. Petersburg Branch of FSUE "STC" Atlas). Since 2009, he became the Director General of FSUE "CentrInform". Dr. Bogdanov is a specialist in cryptographic systems, information security and protection of goods and documents against counterfeiting and tampering. He headed teams of experts for the implementation of government accounting systems of production and turnover of alcohol products; ESUDAP during 2002 to 2005 and EGAIS during 2006 to 2010. Dr. Bogdanov is author of over 100 scientific papers and 16 inventions. In 2004, he was awarded the State Prize of the Russian Federation in the field of science and technology.

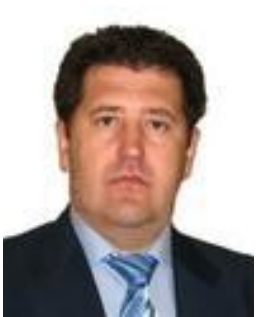

Vitaly Golovko is a Deputy General Director of the State Federal Unitary Enterprise "CentrInform". He graduated with honors from the Higher Naval School of Radioelectronics named A.S Popov with a degree in engineering of automated management systems. In 2000, he joined the development team of "CentrInform". He has participated in projects on the development and implementation of state and corporate information systems of products protection from fakes and counterfeits. He realized the implementation of information system for recording alcohol production in Kazakhstan, the introduction of information systems in Ukraine. He is author of several scientific studies and patents. He is a graduate student of Cherepovets State University in "Data Protection". He is a member of the Advisory Council of the State Duma on the development of pharmaceutical industry in Russia. 


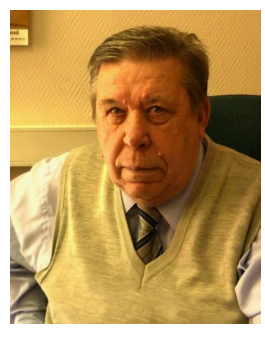

Mikhail Simonov is the Chief Scientific Adviser of the General Director of FSUE "CentrInform". He graduated from the Military Engineering Academy as a radio engineer. He holds a Candidate of Sciences and academic rank of associate professor. Prof. Simonov is a member of the International Academy of Informatization. For a long time, he had been teaching at the Military Academy of Communications and the State University of Telecommunications Bonch-Bruevich. He worked in the CSC "Rainbow" as the Chief Designer of networking and communications systems. In 1998, he joined the engineering team of FSUE "CentrInform".

Prof. Simonov has participated in projects on the development and implementation of state and corporate information systems protection products from fakes and counterfeits. He is also author of over 370 scientific papers, a monograph on "ATM-technology high-speed networks", and 18 patents. In the 2004 he was awarded the State Prize of Russian Federation in the field of science and technology.

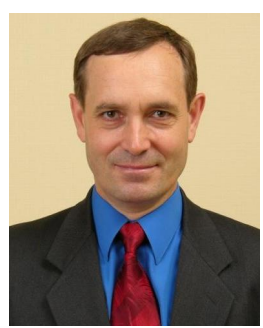

Peter Vikhlyantsev heads the Department of Scientific and Technical Support of FSUE "CentrInform". He was qualified as an engineer at the Military College of Telecommunication connections in 1979 and a research engineer at the Military Academy of Communications in 1985. During 1985 to 1988 , he carried out research lines of immunity to radio communications. He holds a Ph.D. and an academic rank of associate professor. For several years he lectured on communications security and comprehensive data protection. In 2001 he joined the engineering team of FSUE "CentrInform".

Prof. Vikhlyantsev is a member of development and implementation projects of state and corporate information systems of protection products from fakes and counterfeits. He is also author of over 120 scientific papers and 22 patents. 